\title{
Economic evaluation in rheumatology: a necessity for clinical studies
}

Recent advances in the understanding of rheumatoid arthritis (RA) have precipitated a change in its management. It is now clear that $R A$ is not a benign disease. The 'side effects' include joint destruction, loss of functional capacity, frequent work disability, high rates of comorbidity, and an increased mortality rate. ${ }^{1}$ Additionally, patients with a poor prognosis can now be identified at presentation by assessing their level of disease activity (with inflammatory markers and the Health Assessment Questionnaire (HAQ) score), and by looking for the presence of disease specific markers. The latter include rheumatoid factor and genetic markers. ${ }^{2-4}$ The traditional therapeutic approach of NSAIDs followed by second line drugs has produced an unsatisfactory outcome, so now patients with a poor prognosis are being identified for early treatment with intensive drug regimens. These drug costs, which are the main element in the direct costs of care, are significantly greater, but the anticipated trade off will be better long term health. This will facilitate maintenance of employment, avoidance of prosthetic surgery, less reliance on carers, etc, and therefore the indirect costs of lost production, paying for other treatment, and time of carers will be reduced. Allowing for these future savings, the net costs of care may be much less than the direct (gross) costs.

Resources for health care have always been scarce. Managerial and policy arrangements in all the world's developed countries are bringing this into sharp focus. Therefore the greater costs of any improved treatments of rheumatoid arthritis have to be justified. In order to arrive at such justification, and make an unambiguous case for change, economic evaluations of the cost and outcome performances of both new and old treatments will have to be undertaken to identify the gross and net costs of achieving increased patient benefit. It is paramount that these evaluations should be undertaken now, early in the life cycle of these new technologies, before they are widely adopted. It is very difficult to set up effective studies once a treatment has become routine. Similarly, for the cost effectiveness evaluation itself to be cost effective, these evaluations may have to be made prospectively alongside the clinical trials, and not retrospectively as has usually been the case.

The Department of Health Research and Development's research strategy document Research for Health ${ }^{5}$ has encouraged the inclusion of cost effectiveness analysis in trials. Two publications from the NHS ExecutiveImproving the Effectiveness of the NHS ${ }^{6}$ and Improving Clinical Effectiveness ${ }^{7}$-have also focused on this issue.
Furthermore, these papers are encouraging purchasers to purchase only evaluated treatments.

Performing an economic evaluation is not straightforward. Guidelines have been published, including a joint paper by the Department of Health and the Association of the British Pharmaceutical Industry. ${ }^{8}$ Evaluation should involve a multidisciplinary team, including a health economist and a clinician.

Different methods of economic evaluation can be applied. There are four well established methods: cost minimisation analysis, cost effectiveness analysis, cost utility analysis, and cost benefit analysis. The most appropriate method of analysis is determined by the particular question that needs to be answered (table).

- Cost minimisation analysis is used when two treatments with identical, or similar, outcomes are to be compared, for example a comparison of inpatient and outpatient treatment for large joint injections. This is rarely an option, as outcomes usually differ.

- Cost effectiveness analysis is the method of choice when outcomes vary but the effect can be measured in a single dimension, for example cost per improvement in HAQ score or cost per single symptom avoided.

- Cost utility analysis may be appropriate when outcomes vary in more than one dimension. These dimensions may be brought together in a single measure that includes explicit valuation of the various elements to create 'utility' scores. The best known utility measure is the quality adjusted life year (QALY). QALYs are calculated by estimating the total life years gained from a treatment and weighting each year to reflect the quality of life in that year. In turn, quality is assessed by combining information on distress (pain) and disability caused by the illness. This method facilitates comparisons between specialties.

- Cost benefit analysis uses monetary measures for both cost and outcome. Difficulties may arise in valuing the outcomes. One possible method is the 'willingness to pay' model as used by Thompson ${ }^{9}$ in an economic evaluation comparing auranofin with placebo. Respondents were willing to pay $22 \%$ of the monthly household income for a complete cure for arthritis. This proportion did not vary with income.

The four approaches listed above are not always distinct from one another. Studies which use health profiles, or indices, as outcome measures are in reality often a hybrid between cost effectiveness and cost utility analysis.

It is important to determine, at the outset, the viewpoint from which the study is to be performed. Ideally the

Definitions of types of economic evaluation

\begin{tabular}{lll}
\hline Type & Definition & Application \\
\hline Cost minimisation analysis (CMA) & $\begin{array}{l}\text { Finds the least cost programme among those shown to be } \\
\text { of equal benefit }\end{array}$ & $\begin{array}{l}\text { When benefits of alternative drugs/programmes are the } \\
\text { same } \\
\text { Drugs/programmes of similar objective which use same } \\
\text { units of benefit } \\
\text { in physical units. }\end{array}$ \\
$\begin{array}{llll}\text { Computes a C:E ratio for comparison } \\
\text { Measures therapeutic consequences in utility units rather } \\
\text { than in physical units. }\end{array}$ & $\begin{array}{l}\text { Computes a C:U ratio for comparison } \\
\text { Measures benefit in monetary units. Computes a net } \\
\text { pecuniary gain }\end{array}$ & Allows for summarising multiple dimensions into one scale \\
Cost benefit analysis (CBA) & & Campare programmes with different objectives
\end{tabular}


broadest-that is, a societal-viewpoint should be taken. This takes into account all the costs and benefits to society in general. However, the viewpoint is usually narrowed to that of the individual patient, the hospital, the NHS, or the government.

Determining the period over which the economic evaluation should be performed can be difficult. In the treatment of early RA, the end point of the clinical trial may be remission, or 12 months, but at this time the economic benefits are only just accruing. It is therefore important that a 'model' is devised to account for future costs, such as orthopaedic surgery, cost of carers, drugs, etc, so that an accurate, full economic evaluation can be performed of the proposed aggressive treatment regimens in RA patients.

As rheumatologists, we are already measuring outcome using standardised, evaluated disease specific scales, for example the $\mathrm{HAQ}^{10}$ when conducting clinical trials. Such measures are ideal units for cost effectiveness analysis. The Short Form 36 health survey questionnaire (SF 36) ${ }^{11}$ and the Nottingham Health Profile ${ }^{12}$ are general health profiles that also can be used. Interestingly, the best examples of economic analysis in rheumatology thus far are for prophylaxis of gastrointestinal disease. ${ }^{13} 14$

We believe that an economic evaluation should be routinely incorporated into clinical trials for rheumatology, as has tended to be the case for other specialties such as cardiology. ${ }^{15}$ The methodology must be standardised so that meaningful comparisons within rheumatology and across specialties can be made. ${ }^{15}$ Therefore cost effectiveness analysis or cost utility analysis will usually be the most appropriate types of economic evaluation. This will be particularly important with the introduction of new therapeutic approaches and the consequent increased drug costs. Only by routinely assessing the cost and outcome of new and existing therapies will purchasers be persuaded of the effectiveness of rheumatological intervention and be encouraged to invest a greater proportion of their purchasing budget into this specialty.

Rheumatology and Rehabilitation Research Unit,

University of Leeds,

36 Clarendon Road

Leeds LS2 9NZ, United Kingdom

Sheffield Centre for Health and Related Research,

University of Sheffield,

Sheffield, United Kingdom

Correspondence to: Prof P Emery.

1 Pincus T, Callaghan L F. The 'side effects' of rheumatoid arthritis: joint destruction, disability and early mortality. $\mathrm{Br} F$ Rheumatol 1993; 32 (suppl 1): 28-37.

2 Emery P, Salmon M, Bradley $H$, et al. Genetically determined factors as predictors of radiological change in patients with early RA. BMF 1992; 305: 1387-9.

3 Gough A K, Lilley J, Eyre S, Holder R L, Emery P. Genetic typing of patients with inflammatory arthritis at presentation is predictive of patients with inflammatory arthritis at presen
outcome. Arthritis Rheum 1994; 37: 1166-70.

4 Young A, Cox N, Davis P, et al. Treatment patterns over 2 years in 577 patients with rheumatoid arthritis of recent onset. Arthritis Rheum 1994; 37 (suppl 9): S258.

5 Peckham. Research for Health. London: Department of Health, 1993.

6 NHS Management Executive. Improving the effectiveness of the NHS. (EL(94)74.) Leeds: NHS Management Executive, 1994.

7 NHS Management Executive. Improving clinical effectiveness. (EL(93)115.) Leeds: NHS Management Executive, 1993.

8 Guidance on Good Practice in the Conduct of Economic Evaluations of Medicines. London: Association of British Pharmaceutical Industry and the Department of Health, May 1994.

9 Thompson M S. Willingness to pay and accept risks to cure chronic disease. Am $\mathcal{F}$ Publ Health 1986; 76: 392-6.

10 Fries J F. Measurement of patient outcome in arthritis. Arthritis Rheum 1980; 23: $137-45$.

11 Jenkinson C, Coulter A, Wright L. Short form 36 (SF 36) health survey questionnaire: normative data for adults of working age. $B M \mathcal{F} 1993$; 306: $1437-40$.

12 Hunt S M, McEwen J, McKenna S P. Measuring health status: a new tool for clinicians and epidemiologists. I $R$ Coll Gen Pract 1985; 35: 185-8.

13 Edelson J T, Tosteson A N, Sax P. Cost effectiveness of misoprostol for prophylaxis against non-steroidal anti-inflammatory drug induced gastrointestinal bleeding. $¥ A M A$ 1990; 264: 41-7.

14 Ventkover J D, Baker A M, Kaplan $H$. Nabumetone in elderly patients with osteoarthritis: economic benefits versus ibuprofen alone or ibuprofen and osteoarthritis: economic benefits versus ibuprofen
misoprostol. Pharmacoeconomics 1994; 5: 335-42.

15 Szczepura A. Finding a way through the cost and benefit maze. BMF 1994; 309: $1314-5$. 